\title{
Helium abundance and speed difference between helium ions and protons in the solar wind from coronal holes, active regions, and quiet Sun
}

\author{
Hui $\mathrm{Fu}^{1}$, M. S. Madjarska ${ }^{2}$, Bo Li ${ }^{1}$, LiDong Xia ${ }^{1}$, ZhengHua Huang ${ }^{1}$ \\ ${ }^{1}$ Shandong Provincial Key Laboratory of Optical Astronomy and Solar-Terrestrial Environment, Institute of Space Sciences, \\ Shandong University, Weihai, 264209 Shandong, China, fuhui@sdu.edu.cn \\ ${ }^{2}$ Max Planck Institute for Solar System Research, Justus-von-Liebig-Weg 3, 37077, Göttingen, Germany, madjarska@mps.mpg.de
}

Accepted XXX. Received YYY; in original form ZZZ

\begin{abstract}
Two main models have been developed to explain the mechanisms of release, heating and acceleration of the nascent solar wind, the wave-turbulence-driven (WTD) models and reconnection-loop-opening (RLO) models, in which the plasma release processes are fundamentally different. Given that the statistical observational properties of helium ions produced in magnetically diverse solar regions could provide valuable information for the solar wind modelling, we examine the statistical properties of the helium abundance $\left(A_{H e}\right)$ and the speed difference between helium ions and protons $\left(v_{\alpha p}\right)$ for coronal holes $(\mathrm{CHs})$, active regions (ARs) and the quiet Sun (QS). We find bimodal distributions in the space of $A_{H e}$ and $v_{\alpha p} / v_{A}$ (where $v_{A}$ is the local Alfvén speed) for the solar wind as a whole. The $\mathrm{CH}$ wind measurements are concentrated at higher $A_{H e}$ and $v_{\alpha p} / v_{A}$ values with a smaller $A_{H e}$ distribution range, while the AR and QS wind is associated with lower $A_{H e}$ and $v_{\alpha p} / v_{A}$, and a larger $A_{\mathrm{He}}$ distribution range. The magnetic diversity of the source regions and the physical processes related to it are possibly responsible for the different properties of $A_{H e}$ and $v_{\alpha p} / v_{A}$. The statistical results suggest that the two solar wind generation mechanisms, WTD and RLO, work in parallel in all solar wind source regions. In $\mathrm{CH}$ regions WTD plays a major role, whereas the RLO mechanism is more important in AR and QS.
\end{abstract}


Key words: solar wind - Sun: abundances - Sun: activity - methods: observational

\section{INTRODUCTION}

Helium is ranked as the second most abundant element in the Sun and in the solar wind (SW), and it is an important tool in exploring the nature of the solar wind. In particular, the difference in the helium ion and proton properties can help us understand the mechanisms for the release, heating and acceleration of the nascent solar wind (e.g., Marsch et al. 1982a; Neugebauer et al. 1996; Steinberg et al. 1996; Reisenfeld et al. 2001; Kasper et al. 2007, 2012). The abundance of helium $\left(A_{H e}\right)$ and the speed difference between helium ions and protons $\left(v_{\alpha p}\right)$ in the solar wind were extensively studied in the past. The abundance of helium is about 8.5\% in the photosphere (e.g., Grevesse \& Sauval 1998; Asplund et al. 2009). Measurements of the corona above polar coronal holes and surrounding quiet Sun areas showed that $A_{H e}$ is in the range $4 \%-5 \%$ (Laming \& Feldman 2001, 2003). The $A_{H e}$ is usually below $5 \%$ in the solar wind and changes with the solar activity (Ogilvie \& Hirshberg 1974; Feldman et al. 1978). Using data obtained by WIND, Aellig et al. (2001) confirmed this finding and also established that this tendency is more clear for the slow SW. By dividing the solar wind into 25 speed intervals, Kasper et al. (2007, 2012) examined the relationship between the helium abundance and the speed of the solar wind for a whole solar activity cycle, and found a strong correlation between $A_{H e}$ and sunspot numbers for the slowest solar wind.

The speeds of helium ions are usually larger than the proton speeds in the solar wind, although helium ions are heavier than protons. Using data obtained by Helios, Marsch et al. (1982a) analysed the speed difference between helium ions and protons, and found that $v_{\alpha p}$ increases with the solar wind speed. While $v_{\alpha p}$ is close to the local Alfvén wave speed in the fast SW, the average $v_{\alpha p}$ for the slow $\mathrm{SW}$ is close to zero, and $v_{\alpha p}$ in the fast $\mathrm{SW}$ decreases with the increase of the heliocentric distances at almost the same rate as of $v_{A}$. Consequently, these results were confirmed from observations made by Ulysses (Neugebauer et al. 1996; Reisenfeld et al. 2001), Wind (Steinberg et al. 1996), and ACE (Berger et al. 2011).

The plasma release, heating and acceleration mechanisms of the nascent solar wind are a fundamental problem in solar and space physics. Two classes of models, the wave-turbulencedriven (WTD) models (Hollweg 1986; Wang \& Sheeley 1991; Cranmer et al. 2007; Verdini et al. 
2009) and the reconnection loop opening (RLO) models (Fisk et al. 1999; Fisk 2003; Schwadron \& McCo 2003; Woo et al. 2004; Fisk \& Zurbuchen 2006) have been proposed to account for this. The essential difference between the two models is that the plasma escapes directly along open magnetic field lines in the WTD models, whereas in the RLO models the plasma is released by reconnection between open magnetic field lines and closed loops. Waves and turbulence are all important in the two plasma release mechanisms. In the RLO models waves originate in the reconnection process, while waves are generated by photospheric motions in the WTD models (e.g., Fisk 2003; Cranmer et al. 2007; Cranmer 2009; Abbo et al. 2016). Determining which physical mechanisms are at work and/or the extend of the contribution of any of the mechanisms is prerequisite for establishing physically realistic models of the solar wind and the heliosphere (Cranmer 2009).

Generally, the solar wind is categorised by speed. However, the speed is not the only classification criterion of the solar wind (Antiochos et al. 2012; Abbo et al. 2016). The solar wind can also be differentiated by some of its in-situ measured properties, like for instance the charge state (Zhao et al. 2009; Landi et al. 2012; Zhao et al. 2014). As the charge state does not change beyond several solar radii, it carries direct information about the temperature of the source region (Owocki et al. 1983; Buergi \& Geiss 1986). Based on the in-situ properties of the proton number density, proton temperature, magnetic filed strength, and solar wind speed, Xu \& Borovsky (2015) classified the solar wind into four categories, coronalhole-origin plasma, streamer-belt-origin plasma, sector-reversal-region plasma, and ejecta. In addition, on the basis of the above four category classification, Camporeale et al. (2017) developed a solar wind classification algorithm using a Machine Learning algorithm.

The solar wind can also be classified by source regions (Neugebauer et al. 2002; Liewer et al. 2004; Fu et al. 2015, 2017; Zhao et al. 2017a,b). This is reasonable as CHs, ARs, and QS are all regarded as the sources of the solar wind, but their actual contribution is still uncertain. How the solar wind is produced in these regions is also still debatable. It is generally accepted that CHs are the sources of the solar wind (e.g., Krieger et al. 1973; Gosling \& Pizzo 1999). The solar wind can also originate from QS regions (e.g., Woo \& Habbal 2000; Feldman et al. 2005; Fu et al. 2015). Another source region of the solar wind that has been investigated in detail recent years are the edges of active regions. From the comparison of the velocity distributions at $2.5 \mathrm{R}_{\odot}$ and potential field extrapolations using Kitt Peak magnetograms, Kojima et al. (1999) found that low-speed wind regions are associated with large magnetic field expansions originating from area adjacent to ARs. Shortly after Winebarger et al. 
(2001) established the presence of intermittent flows with velocities in the range of 5 $20 \mathrm{~km} \mathrm{~s}^{-1}$ at the edge of an AR using data from the Transition Region and Coronal Explorer (TRACE) in the Fe IX/x $171 \AA$ passband. With the launch of the Hinode spacecraft it became possible to further investigate these regions using imaging and spectroscopic data. Sakao et al. (2007) identified the existence of outflowing plasma at the periphery of ARs in images taken with the X-ray Telescope (XRT) on board Hinode and obtained upward Doppler velocities of $\sim 50 \mathrm{~km} \mathrm{~s}^{-1}$ using the Extreme-ultraviolet Imaging Spectrometer (EIS) on Hinode in an Fe XII line (no wavelength is mentioned in the article). Later, Del Zanna (2008) and Harra et al. (2008) investigated these outflows obtaining upflow Doppler velocities ranging from 5 to $50 \mathrm{~km} \mathrm{~s}^{-1}$ in coronal lines. Bryans et al. (2010) established that the outflows can be present for several days and also identified multiple velocity components in the EIS Fe XII and Fe XIII lines of up to $200 \mathrm{~km} \mathrm{~s}^{-1}$. The follow-up finding of upflows at heights between 1.5 and $2.5 \mathrm{R}_{\odot}$ in the solar atmosphere using data from the Ultra-Violet Coronagraph Spectrometer on board SoHO in the H I Ly $\alpha$ and O vi doublet lines at 1031.9 $\AA$ and 1037.6 ^ (Zangrilli \& Poletto 2012) further supported the evidence that AR peripheries are a possible source of the slow SW. Several studies followed investigating both observationally and through modelling the association of AR upflows with the solar wind (He et al. 2010; van Driel-Gesztelyi et al. 2012; Culhane et al. 2014; Mandrini et al. 2014; Brooks et al. 2015; Galsgaard et al. 2015; Vanninathan et al. 2015; Zangrilli \& Poletto 2016; Baker et al. 2017).

In the present paper, we analyse $A_{H e}$ and $v_{\alpha p} / v_{A}$ (where $v_{A}$ is the local Alfvén speed) of the solar wind for the three general types of solar regions, coronal holes (CHs), active regions (ARs) and the quiet Sun (QS), during three phases of the solar activity cycle. The magnetic field structures are significantly different in these three regions, with CHs generally occupied by large scale open magnetic field lines, whereas AR and QS regions are mainly taken up by closed loops (Wiegelmann \& Solanki 2004; Ito et al. 2010; Wiegelmann et al. 2014). We aim at demonstrating the differences in the properties of $A_{H e}$ and $v_{\alpha p} / v_{A}$ for the three source region solar wind and during three phases of the solar cycle activity. Our expectations are that statistical observational results on the variabilities of $A_{H e}$ and $v_{\alpha p} / v_{A}$ produced in magnetically diverse solar regions may provide helpful and valuable information for the solar wind modelling.

The paper is organised as follows: In Section 2, we describe the data and our method 
of analysis. The statistical results are presented and discussed in Section 3. Section 4 summarises the present study, ending with concluding remarks.

\section{DATA AND ANALYSIS}

The data used in this study are all obtained by the WIND spacecraft. The proton and helium ion velocities and densities were recorded by the Solar Wind Experiment (SWE) Faraday Cup instruments (Ogilvie et al. 1995). The $A_{H e}$ was obtained from the density ratio between the helium ions and protons. The magnetic field was measured by the Magnetic Field Investigation (MFI) (Lepping et al. 1995). The speed uncertainties of the solar wind are less than $0.16 \%$ (Kasper et al. 2006). In some cases, the SWE could not yield accurate helium ion measurements (Steinberg et al. 1996). First, when the proton energy-per-charge distribution is very broad, it makes the helium ion signal overpowered by the proton signal, and thus the helium ion signal cannot be extracted. Second, when the helium ion flux is unusually low, it is down the detection threshold of the detectors. Third, if the solar wind speed is too high, the helium ions may exceed the highest energy-per-charge step of SWE, thus becoming undetectable. In order to ensure accurateness, we only use data that are free of the above mentioned discrepancies. The time resolution of the data is 92 seconds. The data were averaged over $1 \mathrm{hr}$. Generally, the direction of the $v_{\alpha p}$ is assumed along the magnetic field lines (e.g., Asbridge et al. 1976; Marsch et al. 1982a; Steinberg et al. 1996; Berger et al. 2011; Reisenfeld et al. 2001). The $v_{\alpha p}$ was calculated as $v_{a p}=\left(v_{r a}-v_{r p}\right) / \cos (\theta)$, where $v_{r a}$ and $v_{r p}$ are the radial speed of the helium ions and protons, respectively, and $\theta$ is the angle between the radial vector and the magnetic field. In order to reduce the uncertainties, we discarded the observations in which $\theta$ is greater than 72.5 degrees $(\cos (\theta)<0.3)$ as done in Reisenfeld et al. (2001). The $v_{\alpha p}$ is usually compared with the local Alfvén speed $\left(v_{A}\right.$; Marsch et al. 1982a; Steinberg et al. 1996; Berger et al. 2011; Reisenfeld et al. 2001) which was calculated as $v_{A}=22.3 * B_{r t n} / \sqrt{\left(n_{p}+4 * n_{a}\right)}$, where $B_{r t n}, n_{p}$, and $n_{a}$ are the magnetic filed strength in $\mathrm{nT}$, and density of proton and helium ions in number per cubic centimetre (n/cc). At last, $v_{\alpha p}$ was divided by $v_{A}$.

For completeness, we repeat here the description of the two-step mapping procedure (Neugebauer et al. 1998, 2002; Liewer et al. 2004) that was used in tracing the solar wind back to the solar surface (already described in Fu et al. $(2015,2017)$ ). First, each solar wind parcel is traced back in a ballistic approach to the source surface which is implemented by 
the coronal magnetic field model. Second, the wind parcel is traced from the source surface to the photosphere by following the magnetic filed lines computed by a potential field source surface (PFSS) model (Schatten et al. 1969; Altschuler \& Newkirk 1969). The footpoints of the solar wind parcels were then placed on the photospheric magnetograms obtained with the Michelson Doppler imager (MDI, Scherrer et al. 1995) and the EUV images taken by the Extreme-ultraviolet Imaging Telescope (EIT, Delaboudinière et al. 1995) on board Solar and Heliospheric Observatory (SoHO, Domingo et al. 1995). The regions with the located footpoints were then categorised into three groups. The solar wind was named by the three type of source regions they originate from, $\mathrm{CH}, \mathrm{AR}$, or $\mathrm{QS}$ wind.

The categorisation scheme is demonstrated in Figure 1. The footpoint locations are overplotted on the EIT $284 \AA$ images (a1, b1, c1, d1) and MDI magnetograms (a2, b2, c2, d2) marked by red crosses. The classification of the source regions relies on the coronal hole and magnetically concentrated area boundaries. The wind is classified as $\mathrm{CH}$ wind if its footpoints are located within the $\mathrm{CH}$ boundaries. An AR wind is defined if its footpoints fall in a magnetically concentrated area which is a numbered NOAA AR. When a footpoint is positioned out of any $\mathrm{CH}$ and magnetically concentrated area, it is then marked as a QS wind. More details on how the boundaries are determined, as well as the classification of the source regions and the tracing back procedure, can be found in Fu et al. $(2015,2017)$.

The intervals occupied by Interplanetary Coronal Mass Ejections (ICMEs) were discarded where the charge states $\mathrm{O}^{7+} / \mathrm{O}^{6+}$ exceed $6.008 \exp (-0.00578 v)$, where $v$ is the ICME speed in $\mathrm{km} \mathrm{s}^{-1}$ (Richardson \& Cane 2004). The daily averaged solar wind velocity was used in tracing the solar wind back to the source surface, therefore one footpoint for each day is determined. The data used here cover the years from 2000 to 2008. This time period covers the solar maximum (2000 - 2001, hereafter MAX), the decline (2002 - 2006, DEC), and the minimum phases $(2007-2008, \mathrm{MIN})$ of cycle 23 . The statistical results are based on the following number of measurements. For the full speed range (Figure 3), the hourly in-situ samples of the solar wind are 2844, 694, 1634, and 516 for the solar wind as a whole, $\mathrm{CH}$, AR, and QS wind during solar maximum, respectively. The hourly in-situ samples of the solar wind are 6362, 2340, 2282, and 1740, respectively during DEC, and 2169, 635, 352, 1182 during the MIN phase. 

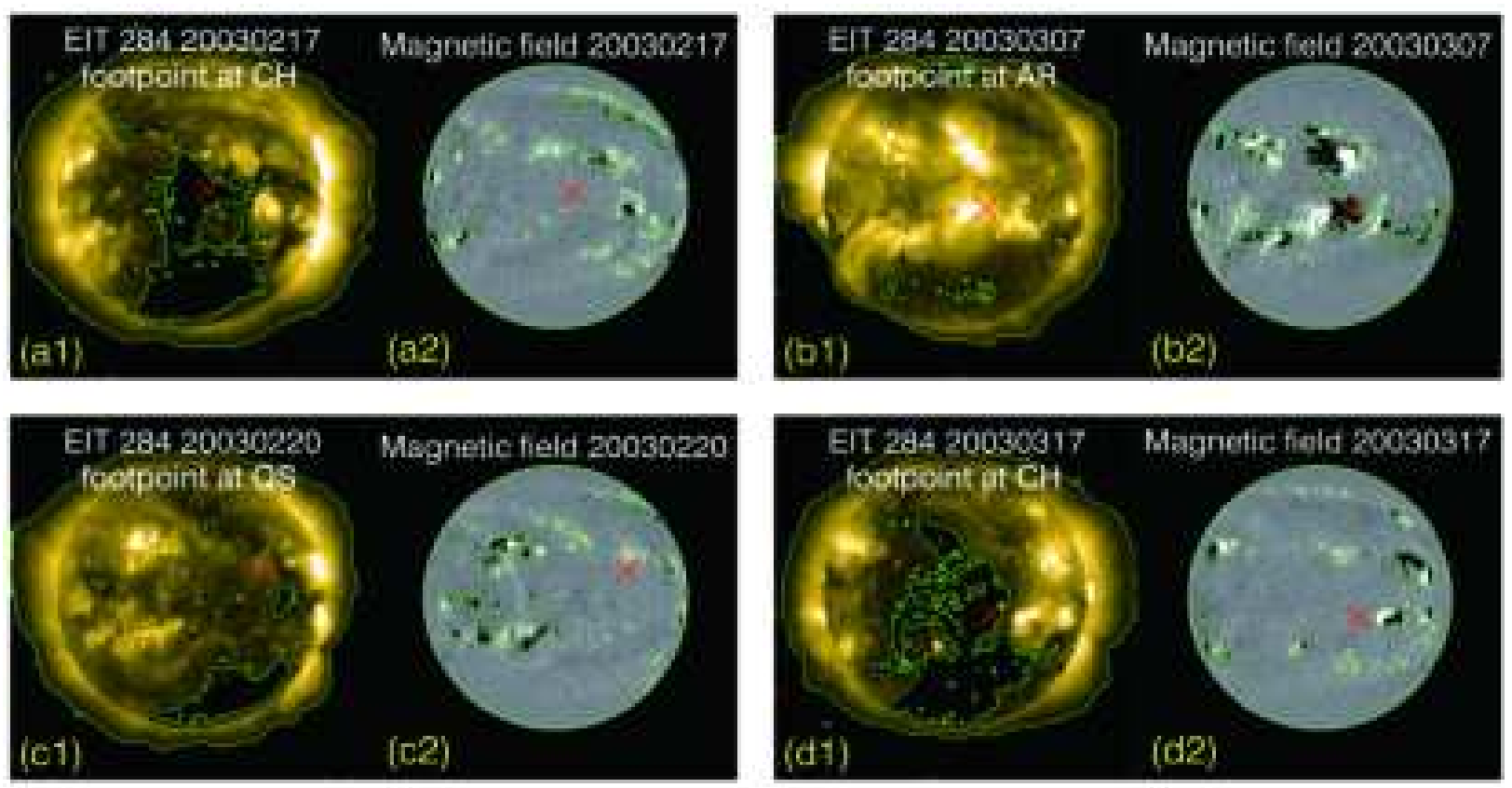

Figure 1. Illustration of the classification scheme of the solar wind. EIT $284 \AA$ images (a1, b1, c1, d1) and corresponding photospheric magnetograms (a2, b2, c2, d2) are shown. The green contours outline the CHs and magnetically concentrated area boundaries. The footpoints are represented by red crosses.

\section{RESULTS AND DISCUSSION}

\subsection{Full speed range solar wind}

Figure 2 presents the scatter (left panel) and contour (right panel) plots of the solar wind measurements in speed, $A_{H e}$, and $v_{\alpha p} / v_{A}$ space. Clearly, the solar wind as a whole is separated into two main parts in the three-dimensional space which is more evident from the contour plots. One part lies in the region of higher $A_{H e}$ and $v_{\alpha p} / v_{A}$, and a wider speed range, coming mainly from CHs. In contrast, the other part has lower values of $A_{H e}, v_{\alpha p} / v_{A}$, and a wider $A_{H e}$ range, that mainly originates from $\mathrm{AR}$ and QS regions. The quantitative analyses of $A_{H e}$ and $v_{\alpha p} / v_{A}$ for the three source region solar wind are given in the following.

Figure 3 (first column) shows the measurements of $A_{H e}$ vs $v_{\alpha p} / v_{A}$ for the solar wind as a whole. The ranges of $A_{H e}(0-10)$ and $v_{\alpha p} / v_{A}(-1.0-1.0)$ are divided into 20 parts. This means that delta $A_{H e}$ and delta $v_{\alpha p} / v_{A}$ are 0.5 and 0.1 , respectively, and the space of $A_{H e}$ and $v_{\alpha p} / v_{A}$ is divided into 400 subsections. We also made estimations of the $A_{H e}$ vs $v_{\alpha p} / v_{A}$ for each individual source region, namely, $\mathrm{CH}$, AR and QS (second, third and fourth columns, respectively) that make the total contribution in the first column of Figure 3 . The results are also obtained for the three solar cycle phases, the MAX, the DEC, and the MIN as the $A_{H e}$ is known to change with the solar cycle activity (Aellig et al. 2001; Kasper et al. 

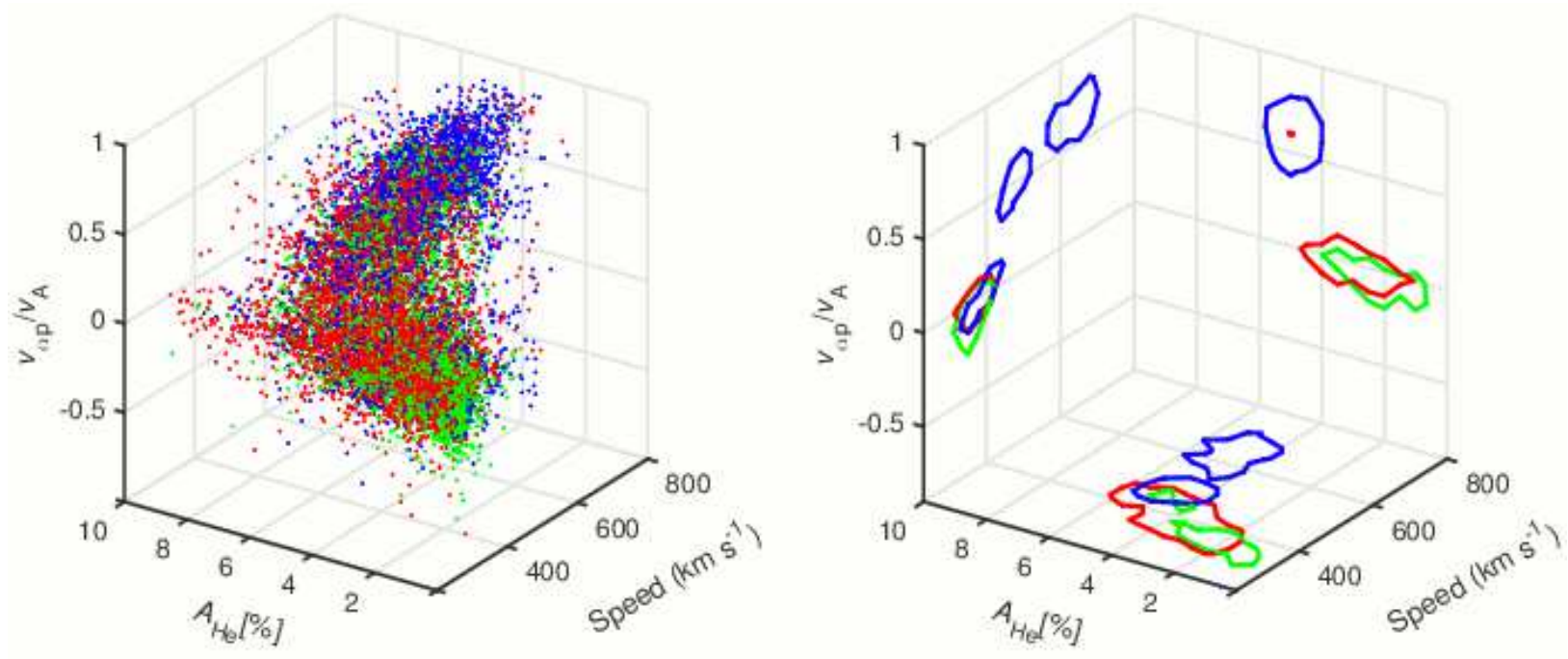

Figure 2. Scatter (left panel) and contour (right) plots of the solar wind in speed, $A_{H e}$, and $v_{\alpha p} / v_{A}$ space. Blue, red, and green represent the wind from $\mathrm{CH}, \mathrm{AR}$, and QS regions, respectively. The contours correspond to $50 \%$ of the maximum counts for different source region solar wind.

2007, 2012). The solar wind as a whole (first column in Figure 3) has a bimodal distribution in the $A_{H e}$ and $v_{\alpha p} / v_{A}$ space. In order to give a quantitative evaluation of the two peaks of the distribution, we estimated the proportions of the count measurements of $A_{H e}$ and $v_{\alpha p} / v_{A}$ averaged over $1 \mathrm{hr}$ located inside the $50 \%$ contour lines of the solar wind as a whole. One of the peaks of this distribution lies in the range of higher $A_{H e}$ and $v_{\alpha p} / v_{A}$ (hereafter H_av) and has proportions of $25 \%, 33 \%$, and $26 \%$ of the total counts for the solar MAX, DEC and MIN. The proportions are given in each panel of Figure 3. In H_av the $A_{H e}$ ranges are $3.75-5.75,3.75-5.75$, and $3.5-5.25$, and the $v_{\alpha p}$ ranges are $0.3-0.7,0.3-0.8$, and 0.2 - 0.7, respectively. In contrast, the other peak covers lower values of $A_{H e}$ and $v_{\alpha p} / v_{A}$ with contributions of 48\%, 27\%, and 34\% during the MAX, DEC, and MIN (hereafter L_av). The corresponding $A_{H e}$ ranges are $1.25-5.50,1.00-3.75$, and $0.25-3.00$, while the $v_{\alpha p}$ ranges are $-0.3-0.2,-0.2-0.1$, and $-0.2-0.1$. It is notable that the $A_{H e}$ distribution ranges of H_av are narrower than the ranges of L_av, 2.0 vs 4.25, 2.0 vs 2.75, and 1.75 vs 2.75, during the MAX, DEC and MIN, respectively. We note that Bourouaine et al. (2011) have also obtained a bimodal distribution in the $A_{H e}$ and $v_{\alpha p} / v_{A}$ space. However, the difference from their study (see their Figure 3) is that we give the distributions for the solar wind originating from various source regions.

We then estimated the count contributions of H_av and L_av for each of the solar wind source regions by applying the same $A_{H e}$ and $v_{\alpha p} / v_{A}$ ranges as estimated from the whole Sun bimodal distribution. A noticeable finding here is that the $\mathrm{CH}$ wind counts are concentrated 


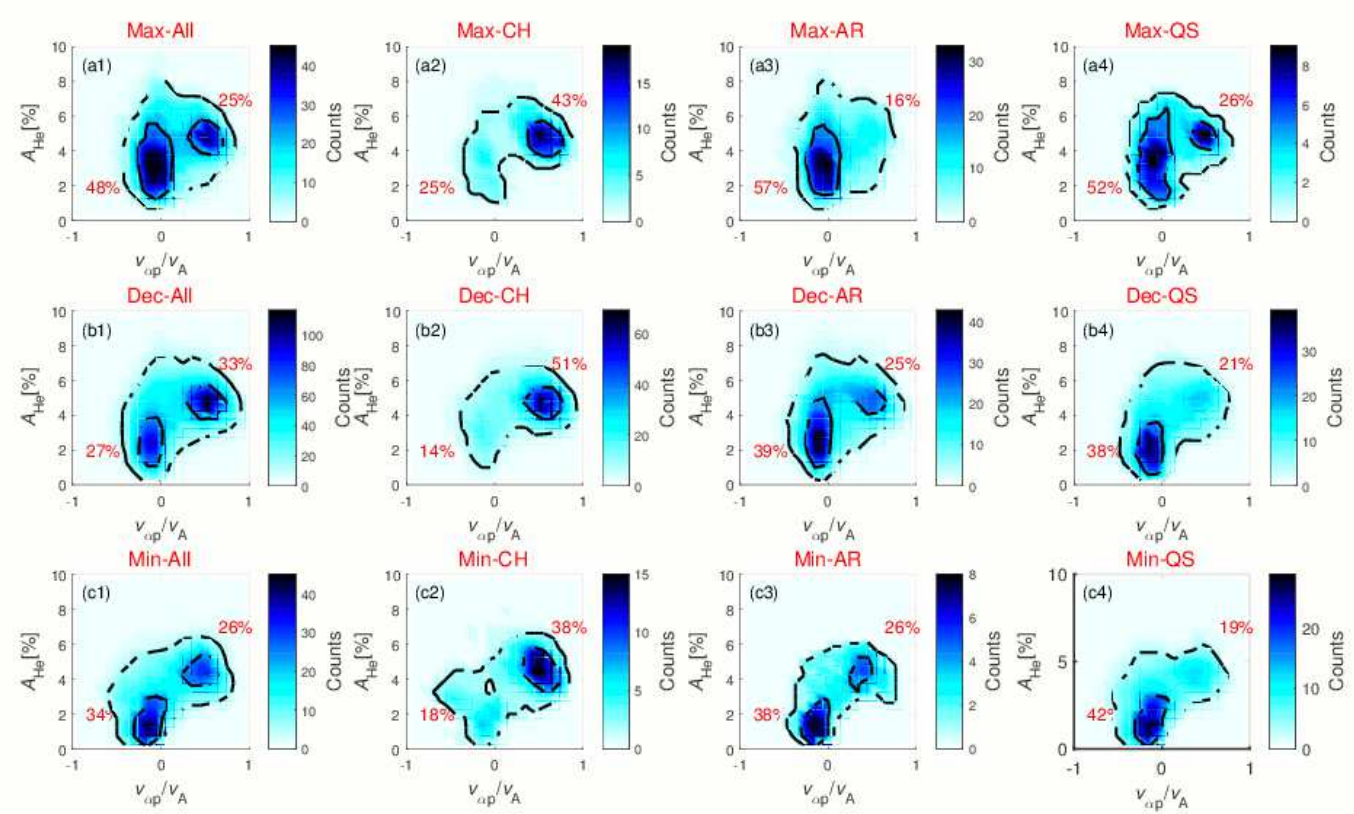

Figure 3. Contour plots of the solar wind originating in $\mathrm{CH}, \mathrm{AR}$, and QS regions in the $A_{H e}$ and $v_{\alpha p} / v_{A}$ space. The first to fourth columns correspond to the wind as a whole, and from $\mathrm{CH}, \mathrm{AR}$, and QS regions, respectively. The top, middle, and bottom panels represent the solar maximum, decline, and minimum phases. The percentages of the samples located in the concentrations of higher $A_{H e}$ and $v_{\alpha p} / v_{A}$ values (H_av) and lower $A_{H e}$ and $v_{\alpha p} / v_{A}$ values (L_av) are also given. The solid line contour corresponds to $50 \%$ and the outer contour to $10 \%$.

in the H_av (Figure 3, second column). The lower limit contributions of H_av are much higher than those of L_av for $\mathrm{CH}$ wind. In contrast, for the AR and QS wind the proportions of L_av are much higher than H_av (Figure 3, third and fourth column). Thus, the AR and the QS wind counts are predominantly located in the L_av.

How the results change if the three types $(\mathrm{CH}, \mathrm{AR}$, and $\mathrm{QS})$ solar wind are determined more restrictively? Usually the footpoints of the solar wind stay in a particular region for a few days (as shown in Figure 1 of Fu et al. (2017)). A boundary wind is defined when the footpoint of the solar wind moves from one region to another (Neugebauer et al. 2002). Here, if the footpoint is located at the same region for more than 2 days, the solar wind associated with the first and last 12 hours, between the change of the footpoint location is defined as a boundary wind. This way only the core wind for a certain region can be extracted. As expected, the samples of the data become smaller. However, the distribution characteristics of the core solar wind are almost the same as in the original selection scheme. The only clear difference is that the proportions of L_av decrease for the $\mathrm{CH}$ wind.

Several studies indicate that the solar wind produced by the WTD mechanism has higher $A_{H e}$ and $v_{\alpha p}$, while the solar wind may have lower $A_{H e}$ and $v_{\alpha p}$ when the RLO mechanism is 
at work. While it is challenging to obtain direct observations of the $A_{H e}$ in the solar corona, first ionisation potential (FIP) bias measurements are more readily available. It is found that generally the FIP bias is higher in AR and QS regions (mainly occupied by closed loops) than in $\mathrm{CH}$ regions (generally taken up by open magnetic field lines) (Widing \& Feldman 2001; Feldman et al. 2005; Brooks \& Warren 2011; Baker et al. 2013). It is believed that the reason for the enrichment of the low FIP ions in the corona and the solar wind is that they are ionized earlier in comparison to high FIP elements. The helium has the highest FIP and remains neutral longest. This results in the enrichment/depletion of low FIP elements/helium because only ions interact with waves (Laming 2012, 2015, 2017). It means that the helium abundance should be inversely proportional to the low FIP bias elements in the corona. Thus, the $A_{H e}$ is higher in open magnetic field structures and lower in closed loops if the above mechanism is valid. The helium abundance for the fast SW coming from large CHs is higher and remarkably stable (Schwenn 2006). In contrast, Rakowski \& Laming (2012) suggested that the helium is depleted in closed loops and the depletion efficiency is higher in larger loops, and lower in smaller loops. Furthermore, from simulations, Laming (2017) showed that the $A_{\mathrm{He}}$ is higher in open magnetic field regions and it is lower in closed loops. Suess et al. (2009) found that the solar wind that comes from big streamers has lower helium abundance. The solar wind can be produced by interchange reconnection in the streamers (Huang et al. 2016). This gives the observational support to the notion that the $A_{H e}$ is lower in the closed loops as streamer structures are composed by very large closed loops.

As already mentioned, the speeds of helium ions are usually larger than protons, although the helium ions are heavier than protons. Generally, it is believed that the helium ions are heated by resonant wave-particle interactions (e.g., Hollweg \& Turner 1978; McKenzie \& Marsch 1982; Isenberg 1984) with waves preferentially heating the heavy ions, making them faster than protons. This means that the wave acceleration is the reason why there is a speed difference between helium ions and protons (Cranmer et al. 2008). The fact that $v_{\alpha p}$ decreases with heliocentric distances (Marsch et al. 1982a; Neugebauer et al. 1996; Reisenfeld et al. 2001) suggests that the speed difference between the helium ions and protons is produced near the Sun. Therefore, it supports the idea that the wave superacceleration of helium ions takes place near the Sun, possibly in the region of acceleration of the solar wind (Neugebauer et al. 1996). For the solar wind that escapes directly along open magnetic field lines, the wave accelerated solar wind starts from the chromosphere, whereas the solar wind released from closed loops is initiated higher in the solar atmosphere. 
It is possible that the solar wind that escapes directly along open magnetic field lines has more time to make a speed difference compared with the wind released from loops. This speculation, however, needs to be further supported by modeling. The above effects indicate that the wind that escapes directly along open magnetic field lines (treated by the WTD models) has larger $v_{\alpha p}$, whereas the solar wind released from closed loops (e.g., RLO models) has smaller $v_{\alpha p}$ (Stakhiv et al. 2016).

However, the $v_{\alpha p}$ will also be affected in the following propagation process. First, it is constrained by various instabilities such as the Alfvén/ion-cyclotron and fast-magnetosonic/whistler instabilities (Bourouaine et al. 2013). Qualitatively, those instabilities are only valid when the $v_{\alpha p}$ nears or exceeds the local Alfvén speed (Li \& Habbal 2000; Verscharen \& Chandran 2013; Lu et al. 2009), therefore they have smaller effect on the solar wind with small $v_{\alpha p} / v_{A}$. Second, collisions can reduce $v_{\alpha p}$ in the solar wind (e.g., Marsch et al. 1982b; Kasper et al. 2008). Bourouaine et al. (2011) found a general inverse relationship between the $v_{\alpha p} / v_{A}$ and collisional age, which means the collisions are important. This should be more significant for the slow SW which has higher collisional age. However, the distribution range in collisional age and $v_{\alpha p} / v_{A}$ space are very large (Figure 2 (b) in Bourouaine et al.,2011) which indicates that $v_{\alpha p}$ may still persist in part of the slow speed SW (speed less than $500 \mathrm{~km} \mathrm{~s}^{-1}$ ). Here, the distributions in $A_{H e}$ and $v_{\alpha p} / v_{A}$ space for the intermediate SW (with speed greater than $400 \mathrm{~km} \mathrm{~s}^{-1}$ and less than $500 \mathrm{~km} \mathrm{~s}^{-1}$ ) demonstrate that a higher $v_{\alpha p} / v_{A}$ at $\sim 0.4$ could still be found in the solar wind whose speed is less than $500 \mathrm{~km} \mathrm{~s}^{-1}$ (see Section 3.2 and Figure 5 below for justifications).

Fu et al. (2017) suggested that the two-peak distribution of $\mathrm{CH}$ wind and the anticorrelation between the speed and $\mathrm{O}^{7+} / \mathrm{O}^{6+}$ can be explained qualitatively by both the WTD and RLO models, implying that the combination of the two classes of mechanisms may be at work (Cranmer 2009). The clear bimodal distribution in space of $A_{H e}$ and $v_{\alpha p} / v_{A}$ for the solar wind as a whole (Figure 2 and the first column of Figure 3) can also result from the interplay of the direct plasma release mechanism along open magnetic field lines treated by the WTD models and via reconnection between closed and open fields in the RLO models. This is consistent with the idea that the WTD and RLO scenarios do not need to be 'mutually exclusive with each other' as suggested by Cranmer (2009) and Abbo et al. (2016).

The distributions in space of $A_{H e}$ and $v_{\alpha p} / v_{A}$ for the solar wind that originates from various source regions (the second to fourth columns of Figure 3) provide additional sup- 
port to the notion that possibly the WTD and RLO mechanisms work together. Spectroscopic observations have shown that there exists a stable outflow at the base of $\mathrm{CH}$ regions (Hassler et al. 1999; Xia et al. 2003, 2004; Tu et al. 2005) and the outflow usually corresponds to concentrations of unipolar magnetic fields (Xia et al. 2003, 2004) where the open magnetic field lines are rooted. On the other hand, closed loops dominate in ARs and QS, and therefore, the solar wind plasma is more likely to be released by magnetic reconnection between closed loops and open magnetic field lines (Neugebauer et al. 2002; Feldman et al. 2005; Harra et al. 2008; He et al. 2010; Zangrilli \& Poletto 2012; van Driel-Gesztelyi et al. 2012; Culhane et al. 2014; Mandrini et al. 2014; Baker et al. 2017).

The low count number at L_av and H_av for the three types solar wind can also be explained reasonably by the above suggestion. For the $\mathrm{CH}$ wind (Figure 3, second column) these L_av measurements could be interpreted as related to magnetic reconnection between open magnetic field lines and loops along $\mathrm{CH}$ boundaries, as well as loops associated with small magnetic bipoles, in particular with the emergence of ephemeral regions. In ARs (Figure 3, third column), a low count H_av is possibly related to small-scale coronal holes adjacent to ARs which are often obscured by overlying large loops coming out of the ARs (e.g., Wang 2017). The domination of L_av in the QS is logical as this region is predominately seeded by closed loops. The existence of open field lines in QS regions explains the presence of H_av counts that are smaller but still significant (Woo \& Habbal 1997; Habbal et al. 1997; Woo \& Habbal 2000).

The observational results for the $A_{H e}$ distribution ranges of H_av and L_av in Figure 3 can be reasonably interpreted as an interplay of the WTD and RLO mechanisms as suggested above. The $A_{H e}$ distribution ranges are smaller for the H_av $(2.00,2.00$, and 1.75 during MAX, DEC, and MIN) compared with the L_av (4.25, 2.75, and 2.75). The simulations by Rakowski \& Laming (2012) have shown that closed loops show a depletion effect on helium ions, and the depletion efficiency is higher in larger loops and lower in smaller loops. Therefore, the distributions of $A_{H e}$ are shifted towards lower values and the distribution ranges are wider for the L_av for regions populated by larger loops (e.g., Wiegelmann \& Solanki 2004; Feldman et al. 2005). 


\subsection{Slow, intermediate, and fast solar wind}

The results for the solar wind in full speed range (Figure 3), show a clear bimodal distribution denoted as H_av and L_av. Generally, the solar wind is divided into two categories, fast and slow SW, and two thresholds are usually chosen, $400 \mathrm{~km} \mathrm{~s}^{-1}$ (e.g. Schwenn 2006) or 500 $\mathrm{km} \mathrm{s}^{-1}$ (e.g. Fu et al. 2015; Stakhiv et al. 2016; Fu et al. 2017). To separate the distribution characteristics of Figure 2, we divided the solar wind into three categories, slow SW (speed of less than $400 \mathrm{~km} \mathrm{~s}^{-1}$ ), intermediate SW (speed greater than $400 \mathrm{~km} \mathrm{~s}^{-1}$ and less than 500 $\mathrm{km} \mathrm{s}^{-1}$ ), and fast SW (greater than $500 \mathrm{~km} \mathrm{~s}^{-1}$ ). To explore the distribution characteristics of the solar wind in different speed ranges for the three source regions, we produced distributions in $A_{H e}$ and $v_{\alpha p} / v_{A}$ space for the slow $\mathrm{SW}$, intermediate $\mathrm{SW}$, and fast $\mathrm{SW}$ given in Figure 4, Figure 5, and Figure 6, respectively.

Figure 4 shows the distributions in $A_{H e}$ and $v_{\alpha p} / v_{A}$ space for the slow SW only. The proportions of L_av are much higher than that of H_av (generally far below 10\%, except for $\mathrm{CHs}$ ). The measurements for the slow SW are mainly concentrated at lower $A_{H e}$ and $v_{\alpha p} / v_{A}$ values with wider $A_{H e}$ distribution ranges for all three types slow SW. In contrast, the fast SW (Figure 6) is mainly distributed at higher $A_{H e}$ and $v_{\alpha p} / v_{A}$ values with narrower $A_{H e}$ distribution ranges for all three types fast SW. The difference in the proportions of H_av and L_av for the intermediate SW are obvious comparing the slow SW (dominant L_av) and fast SW (dominant H_av). H_av and L_av are both present in all three types intermediate SW (Figure 5), with H_av that is dominant during the MAX and DEC phases for the whole SW. In general, the distributions for the intermediate SW are more complex than for the slow SW and fast SW which could be seen in Figure 5, second (CHs), third (ARs), and fourth column (QS). As we suggested, the L_av is produced by the RLO mechanism, whereas the H_av associates with the WTD mechanism. Our results indicate that the slow SW is mainly produced by the RLO mechanism, in contrast the fast SW is mainly generated by the WTD regardless of the source types of the solar wind. The WTD and RLO mechanisms are both present in the intermediate SW, with a different input of each mechanism for the different source regions and during different phases of the solar cycle activity. The L_av for the AR region solar wind is most probably related to AR edge outflows (see Sect. 1) released into the solar wind through magnetic reconnection of the AR loops and open magnetic field lines of adjacent $\mathrm{CHs}$.

Based on in-situ observations, Stakhiv et al. (2016) suggested that the solar wind with 

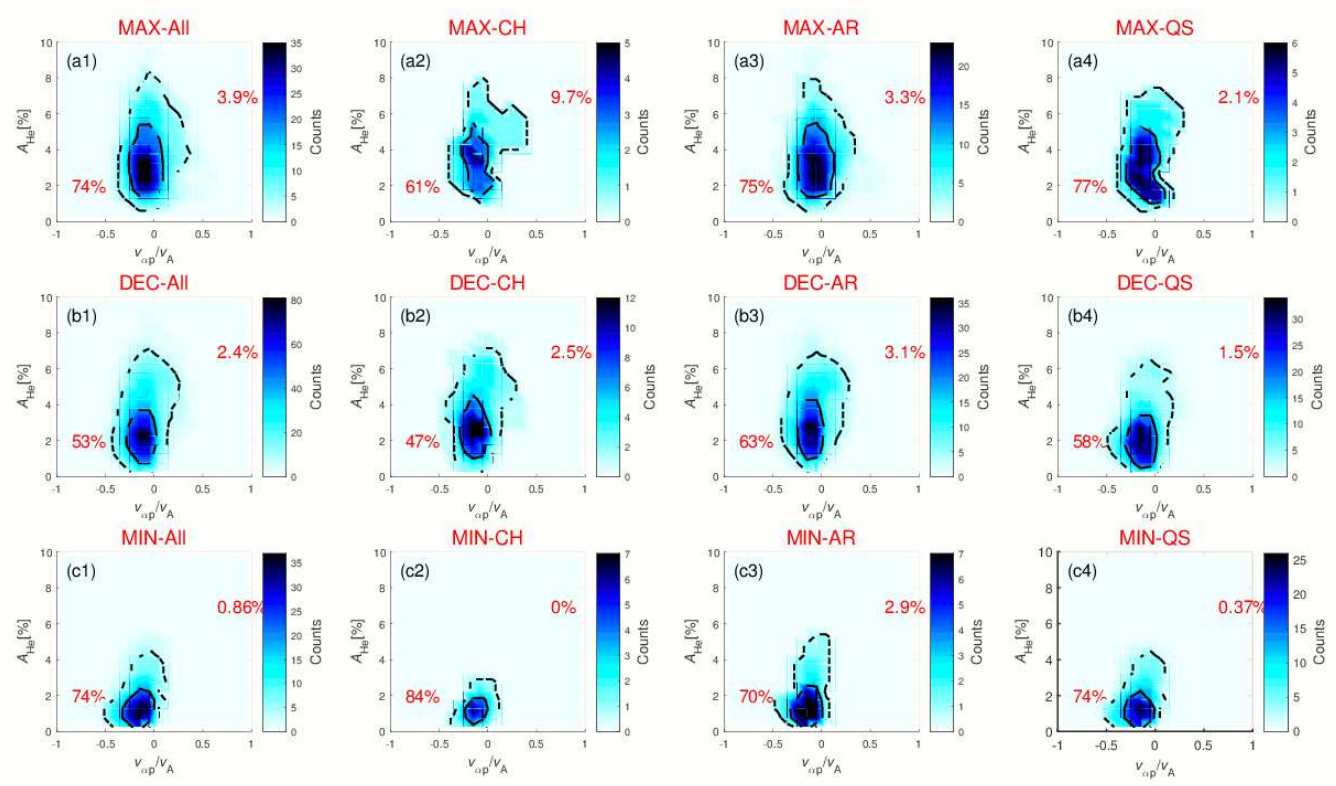

Figure 4. As in Figure 3, but for the slow SW only.

a speed of less than $500 \mathrm{~km} \mathrm{~s}^{-1}$ can be divided into two types, one that is alike the fast SW and the other originating from closed loops. It is clear that the fast SW and "the wind originating from closed loops" in Stakhiv et al. (2016) can be associated with both the WTD and RLO mechanisms. Based on the present statistical results, an interplay of the WTD and RLO mechanisms is present for the solar wind of less than $500 \mathrm{~km} \mathrm{~s}^{-1}$. Therefore, our results give the observational support to the suggestion of Stakhiv et al. (2016).

\section{SUMMARY AND CONCLUDING REMARKS}

The main purpose of the present work was to examine the statistical properties of the $A_{H e}$ and $v_{\alpha p} / v_{A}$ and their distributional characteristics in space of $A_{H e}$ and $v_{\alpha p} / v_{A}$ for three source region solar wind, CHs, ARs and QS. The main results are summarised as follows:

(i) We found bimodal distributions of the solar wind as a whole in the $A_{H e}$ and $v_{\alpha p} / v_{A}$ space. One peak lies in the range of higher values of $A_{H e}$ and $v_{\alpha p} / v_{A}$. In contrast, the other peak is located at lower values of $A_{H e}$ and $v_{\alpha p} / v_{A}$. The analysis for the three source region solar wind shows that the $\mathrm{CH}$ wind counts are concentrated at higher $A_{H e}$ and $v_{\alpha p} / v_{A}$ values with narrower $A_{H e}$ distribution ranges, while the AR and QS wind is mainly located at lower $A_{H e}$ and $v_{\alpha p} / v_{A}$ with larger $A_{H e}$ distribution ranges. 

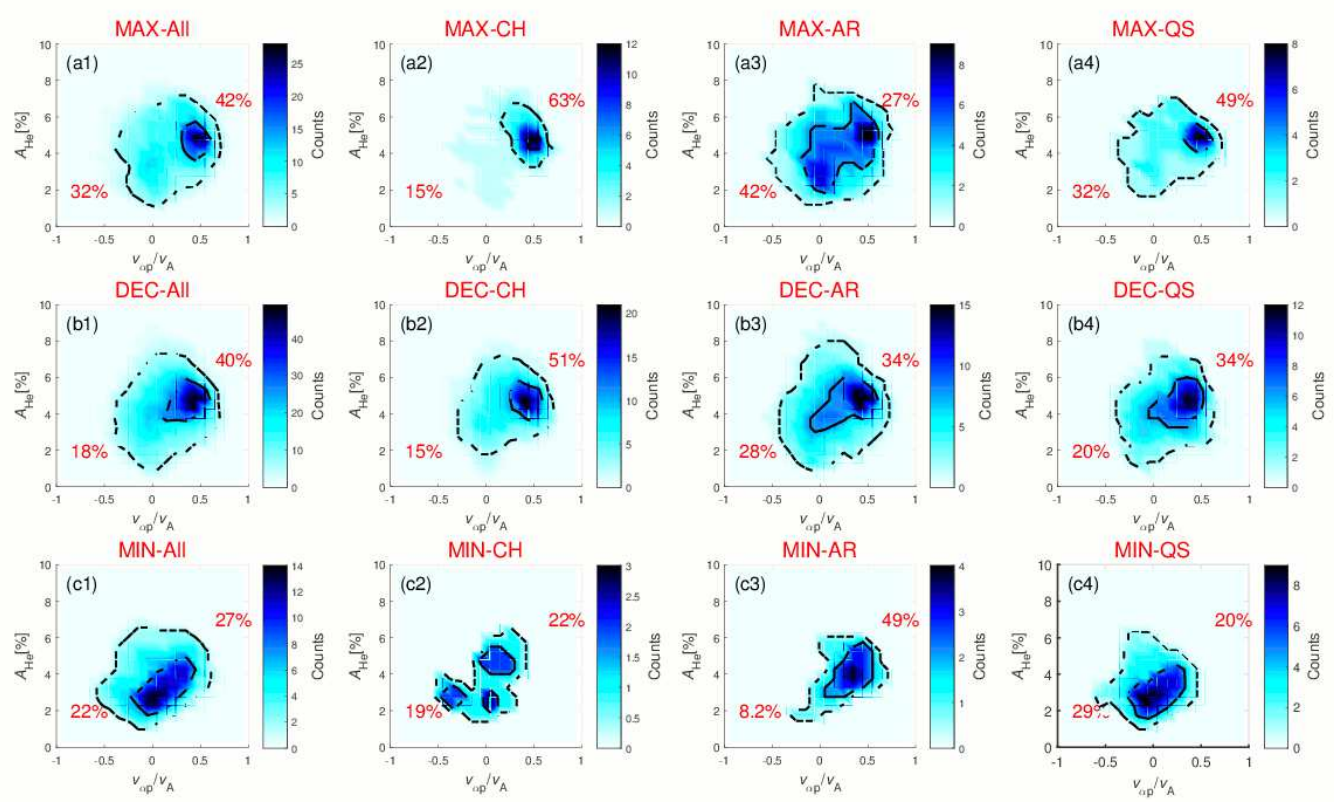

Figure 5. As in Figure 3, but for the intermediate SW only.
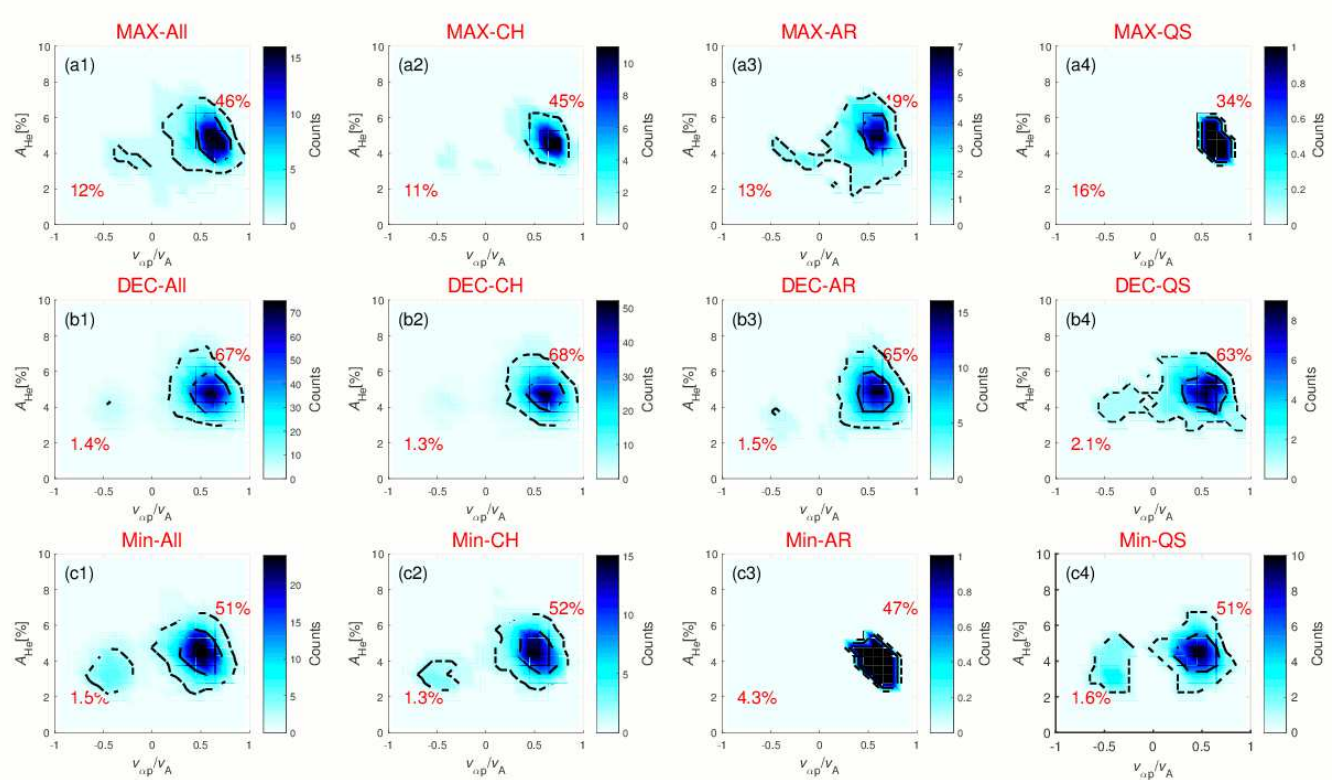

Figure 6. As in Figure 3, but for the fast SW only.

(ii) Almost all of the slow SW (fast SW) measurements are concentrated at lower (higher) $A_{H e}$ and $v_{\alpha p} / v_{A}$ values with wider (narrower) $A_{H e}$ distribution ranges regardless of the source region type. In contrast, the H_av and L_av are both present in all three types $(\mathrm{CH}, \mathrm{AR}$, and QS) intermediate SW. 
The results demonstrate that there are clear differences of $A_{H e}$ and $v_{\alpha p} / v_{A}$ for the three source region solar wind. This indicates that the configuration of the magnetic field has influence on the $A_{H e}$ and $v_{\alpha p} / v_{A}$ properties. We suggest that the two solar wind generation mechanisms, the wave-turbulence-driven (WTD) and the reconnection-loop opening (RLO), work in parallel in all solar wind source regions. In $\mathrm{CH}$ regions WTD plays a major role, whereas RLO is more important in AR and QS regions.

The statistical results for different speed range solar wind indicate that the slow SW (speed less than $400 \mathrm{~km} \mathrm{~s}^{-1}$ ) is mainly produced by the RLO mechanism, in contrast the fast SW (speed greater than $500 \mathrm{~km} \mathrm{~s}^{-1}$ ) is mainly generated by the WTD mechanism regardless of the source types of the solar wind. Whereas both the WTD and RLO mechanisms play role for the generation of the intermediate SW (speed range $400-500 \mathrm{~km} \mathrm{~s}^{-1}$ ).

The future Solar Orbiter mission that comes as close as 0.285 AU to the Sun should help reduce the uncertainties in tracing the solar wind back to the Sun and thus bring more accurate evaluation of the properties of $A_{H e}$ and $v_{\alpha p}$ for different source region solar wind.

\section{ACKNOWLEDGEMENTS}

The authors thank the referee Pascal Demoulin for the very helpful comments and suggestions. Analysis of Wind SWE observations is supported by NASA grant NNX09AU35G. $\mathrm{SoHO}$ is a project of international cooperation between ESA and NASA. This research is supported by the National Natural Science Foundation of China (41604147, 41474150 and 41474149). H.F. thanks the Shandong provincial Natural Science Foundation (ZR2016DQ10). Z.H. thanks Young Scholars Program of Shandong University, Weihai.

\section{REFERENCES}

Abbo L., et al., 2016, Space Sci. Rev., 201, 55

Aellig M. R., Lazarus A. J., Steinberg J. T., 2001, Geophys. Res. Lett., 28, 2767

Altschuler M. D., Newkirk G., 1969, Sol. Phys., 9, 131

Antiochos S. K., Linker J. A., Lionello R., Mikić Z., Titov V., Zurbuchen T. H., 2012, Space Sci. Rev., 172,169

Asbridge J. R., Bame S. J., Feldman W. C., Montgomery M. D., 1976, J. Geophys. Res., 81,2719

Asplund M., Grevesse N., Sauval A. J., Scott P., 2009, ARA\&A, 47, 481

Baker D., Brooks D. H., Démoulin P., van Driel-Gesztelyi L., Green L. M., Steed K., Carlyle J., 2013, ApJ, 778 , 69

Baker D., Janvier M., Démoulin P., Mandrini C. H., 2017, Sol. Phys., 292, 46

Berger L., Wimmer-Schweingruber R. F., Gloeckler G., 2011, Physical Review Letters, 106, 151103

Bourouaine S., Marsch E., Neubauer F. M., 2011, ApJ, 728, L3

Bourouaine S., Verscharen D., Chandran B. D. G., Maruca B. A., Kasper J. C., 2013, ApJ, 777 , L3

MNRAS 000, 1-19 (2018) 
Brooks D. H., Warren H. P., 2011, ApJ, 727, L13

Brooks D. H., Ugarte-Urra I., Warren H. P., 2015, Nature Communications, 6, 5947

Bryans P., Young P. R., Doschek G. A., 2010, ApJ, 715, 1012

Buergi A., Geiss J., 1986, Sol. Phys., 103, 347

Camporeale E., Carè A., Borovsky J. E., 2017, Journal of Geophysical Research (Space Physics), 122, 10

Cranmer S. R., 2009, Living Reviews in Solar Physics, 6, 3

Cranmer S. R., van Ballegooijen A. A., Edgar R. J., 2007, ApJS, 171, 520

Cranmer S. R., Panasyuk A. V., Kohl J. L., 2008, ApJ, 678, 1480

Culhane J. L., et al., 2014, Sol. Phys., 289, 3799

Del Zanna G., 2008, A\&A, 481, L49

Delaboudinière J.-P., et al., 1995, Sol. Phys., 162, 291

Domingo V., Fleck B., Poland A. I., 1995, Sol. Phys., 162, 1

Feldman W. C., Asbridge J. R., Bame S. J., Gosling J. T., 1978, J. Geophys. Res., 83, 2177

Feldman U., Landi E., Schwadron N. A., 2005, Journal of Geophysical Research (Space Physics), 110, A07109

Fisk L. A., 2003, Journal of Geophysical Research (Space Physics), 108, 1157

Fisk L. A., Zurbuchen T. H., 2006, Journal of Geophysical Research (Space Physics), 111, A09115

Fisk L. A., Schwadron N. A., Zurbuchen T. H., 1999, J. Geophys. Res., 104, 19765

Fu H., Li B., Li X., Huang Z., Mou C., Jiao F., Xia L., 2015, Sol. Phys., 290, 1399

Fu H., Madjarska M. S., Xia L., Li B., Huang Z., Wangguan Z., 2017, ApJ, 836, 169

Galsgaard K., Madjarska M. S., Vanninathan K., Huang Z., Presmann M., 2015, A\&A, 584, A39

Gosling J. T., Pizzo V. J., 1999, Space Sci. Rev., 89, 21

Grevesse N., Sauval A. J., 1998, Space Sci. Rev., 85, 161

Habbal S. R., Woo R., Fineschi S., O'Neal R., Kohl J., Noci G., Korendyke C., 1997, ApJ, 489, L103

Harra L. K., Sakao T., Mandrini C. H., Hara H., Imada S., Young P. R., van Driel-Gesztelyi L., Baker D., 2008, ApJ, 676, L147

Hassler D. M., Dammasch I. E., Lemaire P., Brekke P., Curdt W., Mason H. E., Vial J.-C., Wilhelm K., 1999, Science, 283, 810

He J.-S., Marsch E., Tu C.-Y., Guo L.-J., Tian H., 2010, A\&A, 516, A14

Hollweg J. V., 1986, J. Geophys. Res., 91, 4111

Hollweg J. V., Turner J. M., 1978, J. Geophys. Res., 83, 97

Huang J., Liu Y. C.-M., Klecker B., Chen Y., 2016, Journal of Geophysical Research (Space Physics), 121, 19

Isenberg P. A., 1984, J. Geophys. Res., 89, 6613

Ito H., Tsuneta S., Shiota D., Tokumaru M., Fujiki K., 2010, ApJ, 719, 131

Kasper J. C., Lazarus A. J., Steinberg J. T., Ogilvie K. W., Szabo A., 2006, Journal of Geophysical Research (Space Physics), 111, A03105

Kasper J. C., Stevens M. L., Lazarus A. J., Steinberg J. T., Ogilvie K. W., 2007, ApJ, 660, 901

Kasper J. C., Lazarus A. J., Gary S. P., 2008, Physical Review Letters, 101, 261103

Kasper J. C., Stevens M. L., Korreck K. E., Maruca B. A., Kiefer K. K., Schwadron N. A., Lepri S. T., 2012, ApJ, 745,162

Kojima M., Fujiki K., Ohmi T., Tokumaru M., Yokobe A., Hakamada K., 1999, J. Geophys. Res., 104, 16993

Krieger A. S., Timothy A. F., Roelof E. C., 1973, Sol. Phys., 29, 505

Laming J. M., 2012, ApJ, 744, 115

Laming J. M., 2015, Living Reviews in Solar Physics, 12, 2

Laming J. M., 2017, ApJ, 844, 153

Laming J. M., Feldman U., 2001, ApJ, 546, 552

Laming J. M., Feldman U., 2003, ApJ, 591, 1257

Landi E., Alexander R. L., Gruesbeck J. R., Gilbert J. A., Lepri S. T., Manchester W. B., Zurbuchen T. H., 2012, ApJ, 744, 100

Lepping R. P., et al., 1995, Space Sci. Rev., 71, 207

MNRAS 000, 1-19 (2018) 
Li X., Habbal S. R., 2000, J. Geophys. Res., 105, 7483

Liewer P. C., Neugebauer M., Zurbuchen T., 2004, Sol. Phys., 223, 209

Lu Q., Du A., Li X., 2009, Physics of Plasmas, 16, 042901

Mandrini C. H., et al., 2014, Sol. Phys., 289, 4151

Marsch E., Rosenbauer H., Schwenn R., Muehlhaeuser K.-H., Neubauer F. M., 1982a, J. Geophys. Res., 87, 35

Marsch E., Schwenn R., Rosenbauer H., Muehlhaeuser K.-H., Pilipp W., Neubauer F. M., 1982b, J. Geophys. Res., 87, 52

McKenzie J. F., Marsch E., 1982, Ap\&SS, 81, 295

Neugebauer M., Goldstein B. E., Smith E. J., Feldman W. C., 1996, J. Geophys. Res., 101, 17047

Neugebauer M., et al., 1998, J. Geophys. Res., 103, 14587

Neugebauer M., Liewer P. $\quad$ C., Smith E. J., Skoug R. M., Zurbuchen T. H., 2002,

Journal of Geophysical Research (Space Physics), 107, 1488

Ogilvie K. W., Hirshberg J., 1974, J. Geophys. Res., 79, 4595

Ogilvie K. W., et al., 1995, Space Sci. Rev., 71, 55

Owocki S. P., Holzer T. E., Hundhausen A. J., 1983, ApJ, 275, 354

Rakowski C. E., Laming J. M., 2012, ApJ, 754, 65

Reisenfeld D. B., Gary S. P., Gosling J. T., Steinberg J. T., McComas D. J., Goldstein B. E., Neugebauer M., 2001,

J. Geophys. Res., 106, 5693

Richardson I. G., Cane H. V., 2004, Journal of Geophysical Research (Space Physics), 109, A09104

Sakao T., et al., 2007, Science, 318, 1585

Schatten K. H., Wilcox J. M., Ness N. F., 1969, Sol. Phys., 6, 442

Scherrer P. H., et al., 1995, Sol. Phys., 162, 129

Schwadron N. A., McComas D. J., 2003, ApJ, 599, 1395

Schwenn R., 2006, Space Sci. Rev., 124, 51

Stakhiv M., Lepri S. T., Landi E., Tracy P., Zurbuchen T. H., 2016, ApJ, 829, 117

Steinberg J. T., Lazarus A. J., Ogilvie K. W., Lepping R., Byrnes J., 1996, Geophys. Res. Lett., 23, 1183

Suess S. T., Ko Y.-K., von Steiger R., Moore R. L., 2009, Journal of Geophysical Research (Space Physics), 114, A04103

Tu C.-Y., Zhou C., Marsch E., Xia L.-D., Zhao L., Wang J.-X., Wilhelm K., 2005, Science, 308, 519

Vanninathan K., Madjarska M. S., Galsgaard K., Huang Z., Doyle J. G., 2015, A\&A, 584, A38

Verdini A., Velli M., Buchlin E., 2009, Earth Moon and Planets, 104, 121

Verscharen D., Chandran B. D. G., 2013, ApJ, 764, 88

Wang Y.-M., 2017, ApJ, 841, 94

Wang Y.-M., Sheeley Jr. N. R., 1991, ApJ, 372, L45

Widing K. G., Feldman U., 2001, ApJ, 555, 426

Wiegelmann T., Solanki S. K., 2004, Sol. Phys., 225, 227

Wiegelmann T., Thalmann J. K., Solanki S. K., 2014, A\&ARv, 22, 78

Winebarger A. R., DeLuca E. E., Golub L., 2001, ApJ, 553, L81

Woo R., Habbal S. R., 1997, Geophys. Res. Lett., 24, 1159

Woo R., Habbal S. R., 2000, J. Geophys. Res., 105, 12667

Woo R., Habbal S. R., Feldman U., 2004, ApJ, 612, 1171

Xia L. D., Marsch E., Curdt W., 2003, A\&A, 399, L5

Xia L. D., Marsch E., Wilhelm K., 2004, A\&A, 424, 1025

Xu F., Borovsky J. E., 2015, Journal of Geophysical Research (Space Physics), 120, 70

Zangrilli L., Poletto G., 2012, A\&A, 545, A8

Zangrilli L., Poletto G., 2016, A\&A, 594, A40

Zhao L., Zurbuchen T. H., Fisk L. A., 2009, Geophys. Res. Lett., 36, L14104 
Zhao L., Landi E., Zurbuchen T. H., Fisk L. A., Lepri S. T., 2014, ApJ, 793, 44

Zhao L., Landi E., Lepri S. T., Kocher M., Zurbuchen T. H., Fisk L. A., Raines J. M., 2017a, ApJS, 228, 4

Zhao L., Landi E., Lepri S. T., Gilbert J. A., Zurbuchen T. H., Fisk L. A., Raines J. M., 2017b, ApJ, 846, 135 van Driel-Gesztelyi L., et al., 2012, Sol. Phys., 281, 237

This paper has been typeset from a $\mathrm{T}_{\mathrm{E}} \mathrm{X} / \mathrm{LAT} \mathrm{EX}$ file prepared by the author. 